\title{
Análisis del Mecanizado en herramientas de acero rápido recubiertas con nitruro de vanadio
}

\section{Machining analysis on speed steel tools coated with vanadium nitride}

\section{Análise da usinagem em ferramentas de aço rápido recobertas com nitreto de vanádio}

\author{
Jesús David Villarreal-López ${ }^{1}$, William Arnulfo Aperador-Chaparro², Jairo Rafael Cortes-Lizarazo ${ }^{3}$
}

Forma de citar: J. D. Villarreal-López, W. A. Aperador-Chaparro, J. R. Cortes-Lizarazo, “Análisis del Mecanizado en herramientas de acero rápido recubiertas con nitruro de vanadio”, Respuestas, vol. 21, no. 2, pp. 103-111, 2016.

Recibido:

Febrero 9 de 2016

Aceptado: Mayo 25 de 2016

${ }^{1}$ Maestría en Mecatrónica david042_7@hotmail.com orcid: 0000-0001-7097-6314 Facultad de Ingeniería Mecánica,

Universidad Santo Tomas Bogotá-Colombia

${ }^{2}$ Doctorado En Ingeniería william.aperador@unimilitar. edu.co orcid: 0000-0001-9006-0020

Facultad de Ingeniería

Universidad Militar Nueva Granada

Bogotá-Colombia

${ }^{3}$ Maestría en Ingeniería Mecatrónica jairo.rafael.cortes@hotmail.com orcid: 0000-0001-5816-6757

Facultad de Ingeniería

Universidad Militar Nueva

Granada

Bogotá-Colombia

\section{Resumen}

Mediante la técnica de deposición física fase vapor (PVD) usando el método por magnetrón sputtering, se logró el recubrimiento en forma de monocapas de nitruro de vanadio sobre herramientas de acero rápido ASSAB $17 ®$ usados para el mecanizado por arranque de viruta en probetas de acero UNS G1020 para operaciones de cilindrado. Se realizaron cambios progresivos en los parámetros de corte tomando datos de temperatura sobre la herramienta, la probeta y el material removido para su posterior análisis y comparación con los datos obtenidos del mismo proceso realizado con herramientas sin ningún recubrimiento. Subsiguientemente se hizo la caracterización morfológica del recubrimiento mediante microscopio electrónico de barrido a las herramientas de corte. Se cuantificó la rugosidad de cada pobreta, característica fundamental para observar el aumento de la vida útil de la herramienta de corte y la reducción en los tiempos y costos de producción. Los resultados fueron contundentes, justificados en el incremento del desempeño en el arranque de material, mejor transferencia energética en el corte superior acabado superficial en las probetas.

Palabras clave: Herramienta, mecanizado, vida útil.

\begin{abstract}
Using the physical vapor deposition (PVD) technique with magnetron sputtering, we achieved monolayer-coating with vanadium nitride on ASSAB 17TM high speed steel tools used for machining UNS G1020 steal probes in turning operations by chip removal. We performed progressive changes in the cutting parameters, recording data related to temperature of the cutting tool, the graduated cylinder and the removed material for later analysis and comparison with similar data from the process performed with non-coated tools. Subsequently the morphologic characterization of the coating was carried out using scan electron microscope on the cutting tools. Graduate cylinder roughness was quantified to observe the lifetime extension and reduction of production cost reduction derived from the use of coated tools. The results were conclusive, justified in the increased performance in material removal, enhanced energetic transfer during cut and improved surface finished in the probes.
\end{abstract}

Keywords: tool, machining; useful life. 


\begin{abstract}
Resumo
Através da técnica de deposição física fase vapor (PVD) usando o método por pulverização catódica de magnétron, se conseguiu o recobrimento em forma de monocamadas de nitreto de vanádio sobre ferramentas de aço rápido ASSAB 17® usados para a usinagem em provetas de aço UNS G1020 para operações de cilindrado. Realizaram-se mudanças progressivas nos parâmetros de corte tomando dados de temperatura sobre a ferramenta, a proveta e o material removido para sua posterior análise e comparação com os dados obtidos do mesmo processo realizado com ferramentas sim nenhum recobrimento. Subsequentemente, se fez a caracterização morfológica do recobrimento através de microscópio electrónico de varredura nas ferramentas de corte. Qualificou-se a rugosidade de cada proveta, característica fundamental para observar o aumento da vida útil da ferramenta de corte e a redução nos tempos e custos de produção. Os resultados foram contundentes, justificados no incremento do desempenho na maquinagem do material, melhor transferência energética no corte superior do acabado superficial nas provetas.
\end{abstract}

Palavras-chave: ferramenta, usinagem, vida útil.

\section{Introducción}

En toda empresa manufacturera a nivel nacional e internacional existe la preocupación por mantener la calidad de los productos y mejorar tiempos de producción, esto sin incrementar costos de operación, donde quizás es el fenómeno de desgaste presente en las herramientas de corte el principal inconveniente a vencer [1]. Para lograr este objetivo la comunidad científica ha desarrollado la forma de aumentar la resistencia al desgaste en las herramientas de corte que ocasiona una disminución en costos de producción puesto que esto tendría un menor costo que el remplazo total de la herramienta [2].
Colombia al ser un país en vía de desarrollo debe contar en su industria con procesos de manufactura óptimos, los cuales se logran fácilmente teniendo herramientas con mejores propiedades mecánicas que son más duraderas, haciendo que bajen los costos de producción e introduciendo un nuevo concepto llamado manufactura ágil. [3], [4].

Diversas investigaciones a nivel académico han tenido como objetivo el estudio de esta problemática [5] y se han empeñado en generar soluciones a este fenómeno para que poco a poco las industrias de todo tipo de ámbito económico puedan reducir costos, tiempos de operación y fabricación, y aumentos de la calidad de los productos fabricados. [6], [7], [8].

Los recubrimientos duros han sido los desarrollos investigativos con mejores resultados frente a esta problemática [9], estos se concentran en mejorar propiedades mecánicas sobre el material aplicado. Propiedades como tenacidad, resistencia a la oxidación, resistencia al desgaste y coeficiente de fricción [10] [11] son algunas que se mejoran al momento de aplicar recubrimientos sobre aceros pero este desempeño está ligado fuertemente al tipo de técnica implementada al momento de realizar este proceso [12].

La investigación más relacionadas con el tema es donde se obtuvieron capas VN sobre acero AISI 4140 depositadas por medio de la técnica magnetrón sputtering, sobre blancos de pureza de vanadio y hafnio, como resultado de esta investigación se obtuvo que después de aplicarle al acero 4140 el proceso de nitruración de $\mathrm{V}$ la superficie es homogénea 
sin imperfecciones, lo cual nos indica que el elemento posee ahora una mejor resistencia al desgaste [13].

Una vez realizada la revisión bibliográfica se encuentra que no hay investigaciones realizadas acerca del desempeño de las monocapas de $\mathrm{VN}$ en procesos de manufactura por corte de metal, dicha inquietud presenta una oportunidad de investigación en este tema en particular que va a ser la evaluación de la resistencia al desgaste adhesivo de monocapas de $\mathrm{VN}$ depositados en buriles de acero rápido mediante la técnica de deposición física fase vapor (PVD) [14].

El presente trabajo pretende dar a conocer el desempeño y aumento de la vida útil en las herramientas de corte más utilizadas en procesos de arranque de viruta que son los buriles ASSAB $17{ }^{\circledR}$ que fueron recubiertos por monocapas de Nitruro de Vanadio (VN).

\section{Materiales y métodos}

\section{A. Herramientas de corte}

El recubrimiento de monocapas de nitruro de vanadio fue depositado sobre herramientas de acero rápido ASSAB $17 \AA$, que poseen altos niveles de dureza (70HRC) y buena resistencia al desgaste a altas temperaturas. $\mathrm{Su}$ gran valor en dureza es resultado de ser tres veces revenidos los cuales los hacen especiales para producción en serie. Por otra parte, para garantizar homogeneidad en la toma de resultados, las herramientas de corte antes de la deposición de los recubrimientos fueron sometidas a un proceso de afilado mediante un equipo de afilado universal y así garantizar un mismo ángulo al momento de realizar los procesos de arranque de viruta.

\section{B. Recubrimiento con VN.}

La deposición física fase vapor (PVD) es un proceso electroquímico consiste en evaporar un metal puro en la cámara del reactor donde previamente se obtiene un grado de vacío $(1,2$ $\mathrm{X} 10^{-4} \mathrm{mbar}$ ), de esta forma el metal evaporado en contacto con el gas Nitrógeno reactivo forma el compuesto que es aplicado contra la pieza a recubrir, dicha pieza previamente es calentada alrededor de $250^{\circ} \mathrm{C}$ con el objetivo de conseguir un grado de adherencia aceptable de la capa. [9]

Tabla I. Parámetros de deposición utilizados para la obtención de monocapas de NV sobre herramientas de corte.

\begin{tabular}{|c|c|}
\hline Parámetros & Nitruro de Vanadio (VN) \\
\hline Blanco & $\mathrm{V}(99.99 \%)$ \\
\hline Densidad de potencia $\left(\mathrm{W} / \mathrm{cm}^{2}\right)$ & 5,1 \\
\hline Relación $\mathrm{N}_{2} / \mathrm{Ar}(\%)$ & $20 / 80$ \\
\hline Flujo $\mathrm{N}_{2}(\mathrm{SCCM})$ & 10 \\
\hline Presión de trabajo $(\mathrm{mbar})$ & $1,2 \times 10^{-2}$ \\
\hline Voltaje de polarización $(\mathrm{V})$ & -30 \\
\hline Temperatura $\left({ }^{\circ} \mathrm{C}\right)$ & 250 \\
\hline Distancia Blanco-substrato $(\mathrm{cm})$ & 7 \\
\hline Tasa de deposición $(\mathrm{nm} / \mathrm{h})$ & 180 \\
\hline
\end{tabular}

Fuente: [13]

\section{Caracterización morfológica del recubrimiento}

Mediante la microscopía electrónica de barrido se obtienen imágenes tridimensionales permitiendo observar y caracterizar morfológicamente la superficie de la herramienta de corte, la metodología usada fue primero tomar imágenes de la herramienta antes del proceso de mecanizado Figura 1 (a) y después compararlas con las imágenes tomadas después de las pruebas de mecanizado Figura 1 (b), la resolución de toma de muestras está en un rango de $500 \mu \mathrm{m}$. 


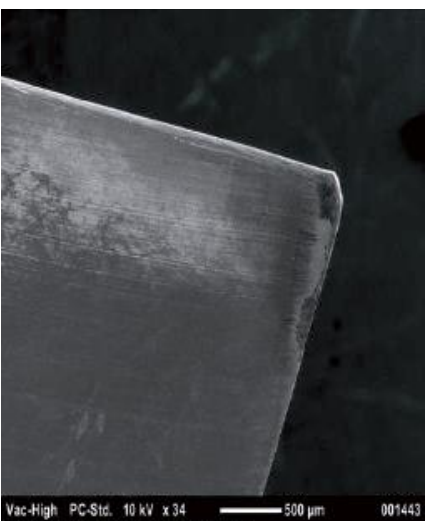

(a)

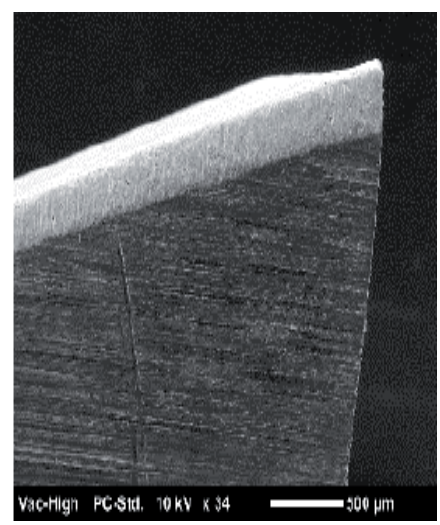

(b)

Figura 1. Micrografía con aumento $34 \mathrm{X}$ de las herramientas de corte recubiertas con VN (a) antes de cilindrado (b) después del cilindrado.

Fuente: Autores

\section{Toma de muestras y procesos de mecanizado}

El montaje realizado en el proceso de mecanizado expuesto en la Figura 2, muestra el material a mecanizar, el cual es una de las 24 barras de acero ASI-SAE 1020 (UNS G1020) de $50 \mathrm{~cm}$ de largo y 1/2" de diámetro. Igualmente, en un portaherramientas se hace el montaje del buril; además, para la obtención de los datos de temperatura se implementó un sensor infrarrojo PSC-CS, que como características principales se tiene que cuenta con un rango de operación de $-40^{\circ} \mathrm{C}$ hasta $1030^{\circ} \mathrm{C}$, asimismo con la implementación de Labiew, se puede observar la variación de medición de los datos del sensor, y el valor promedio de la temperatura. El sensor tiene una frecuencia de $100 \mathrm{~Hz}$, pero debido al procesamiento de labview se están captando
Todas estas características del sensor son favorables para la toma de muestras de temperatura en los tres puntos del sistema que son en el material a mecanizar, en la herramienta de corte y por último en el material removido generado por la operación de desbaste.

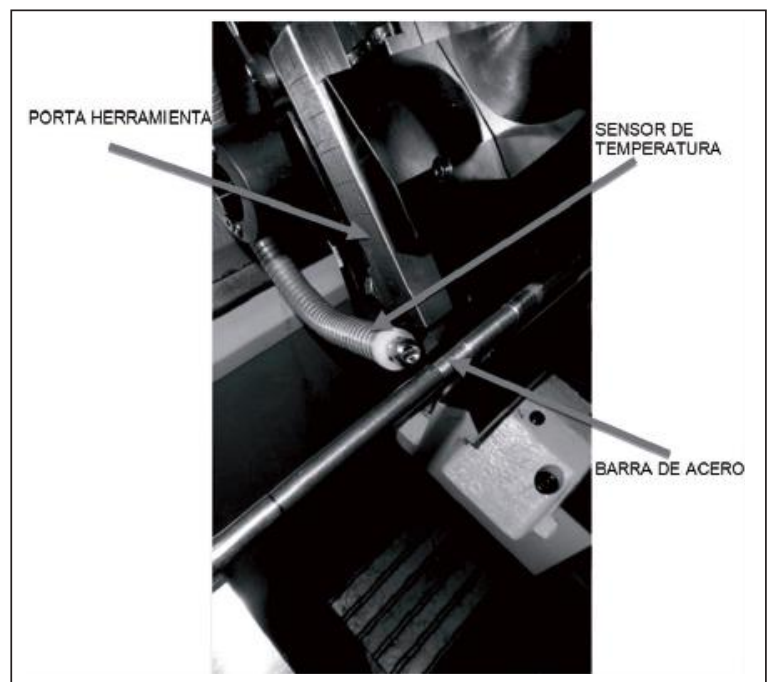

Figura 2. Montaje de probeta y de sensor óptico de temperatura en la máquina de control numérico para operaciones de cilindrado. Fuente: Autores

En una máquina de control numérico para operaciones de cilindrado se programa con los parámetros de corte unos para desbaste y otros para pulido, como se muestra en la siguiente tabla II.

Tabla II. Parámetros de corte

\begin{tabular}{|l|c|c|}
\hline VARIABLE & DESBASTE & PULIDO \\
\hline $\begin{array}{l}\text { Profundidad } \\
\text { de corte }\end{array}$ & $2 \mathrm{~mm}$ & $0,1 \mathrm{~mm}$ \\
\hline $\begin{array}{l}\text { Longitud de } \\
\text { corte }\end{array}$ & $300 \mathrm{~mm}$ & $300 \mathrm{~mm}$ \\
\hline Avance & $0,25 \mathrm{~mm} / \mathrm{rev}$ & $0,05 \mathrm{~mm} / \mathrm{rev}$ \\
\hline \multicolumn{3}{|c|}{ Fuente: autores }
\end{tabular}




\section{E. Rugosidad}

En todo proceso de mecanizado por arranque de viruta, se presenta el fenómeno de rugosidad mecánica. La rugosidad mecánica es el conjunto de irregularidades que posee una superficie producto de las huellas de la

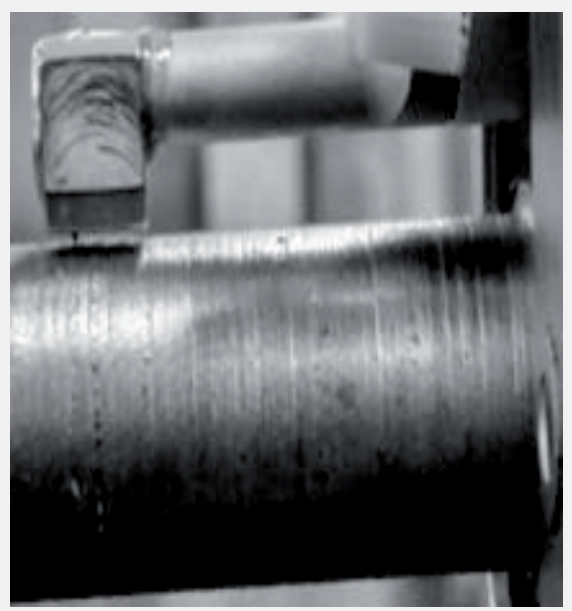

(a) herramienta empleada para la fabricación de la pieza. [11].

Para el cálculo de la rugosidad de las probetas mecanizadas, en la Figura 3, se observa el montaje del rugosímetro mitutoyo referencia sj-310 usado en la prueba.

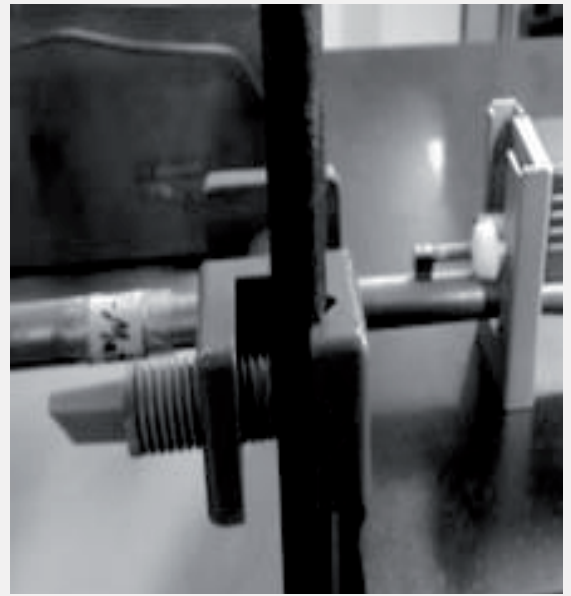

(b)

Figura 3. a) Palpador de Rugosidad; b) Montaje Cálculo de Rugosidad. Fuente; Autores

\section{Resultados y análisis}

\subsection{Análisis de rugosidad}

Se evidencia en la tabla III que el mejor acabado lo produce la herramienta recubierta con monocapas de nitruro de vanadio, esto es gracias a que los recubrimientos duros aumentan la propiedad de autolubricación de elemento sobre el cual fue depositado. [12].

Tabla III. Valores obtenidos de Rugosidad Media

\begin{tabular}{|c|c|}
\hline Tipo de herramienta & Rugosidad $\mathrm{R}_{\mathrm{a}}(\mu \mathrm{m})$ \\
\hline Sin recubrimiento & 3 \\
\hline Nitruro de Vanadio & 1,28 \\
\hline
\end{tabular}

Fuente: Autores

Este resultado de menor rugosidad utilizando herramientas recubiertas es consecuencia de menores temperaturas de operación en el proceso de cilindrado; en las siguientes graficas de temperatura vs tiempo no solo se muestra que se disminuye la temperatura de operación, sino que también la mantiene constante por más tiempo, de allí el mejor acabado superficial uniforme en la probeta.

\subsection{Análisis de los resultados obtenidos de las temperaturas}

Una vez obtenidos los históricos, se procede a graficar los datos de temperatura contra tiempo donde se ve el comportamiento de los dos tipos de herramientas mientras se va desgastando progresivamente manifestado con el aumento de temperatura al transcurrir la prueba; esto se observa en la Figura 4, Figura 5 y Figura 6, mostradas a continuación: 
Vol. 21

No. 2

Jul - Dic 2016

ISSN 0122-820X

E-ISSN 2422-5053

PP: 103-111

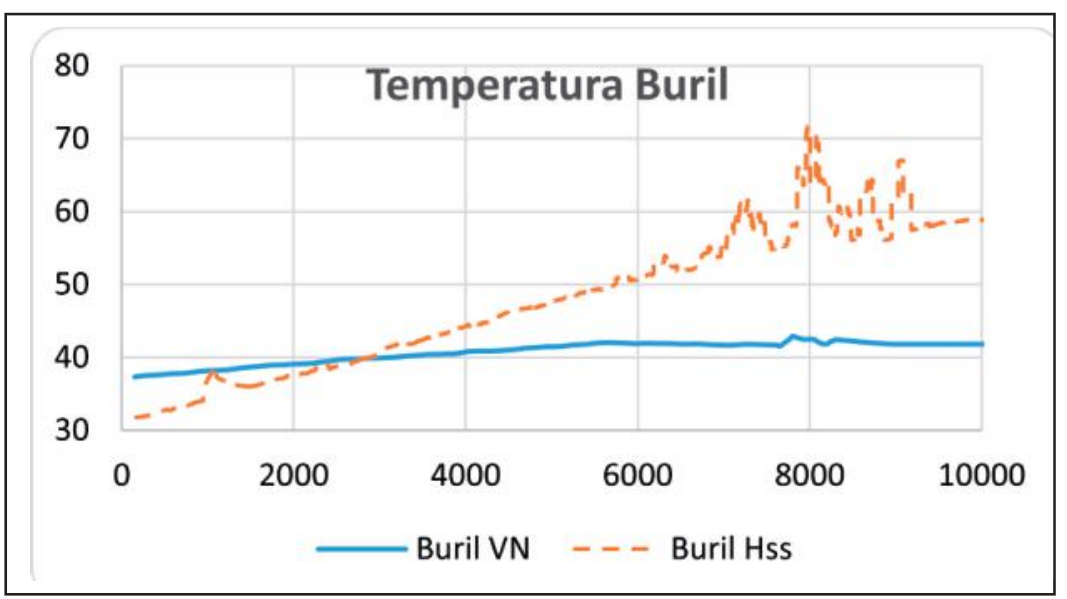

Figura. 4. Temperatura en herramienta con y sin recubierto de VN.

Fuente: autores

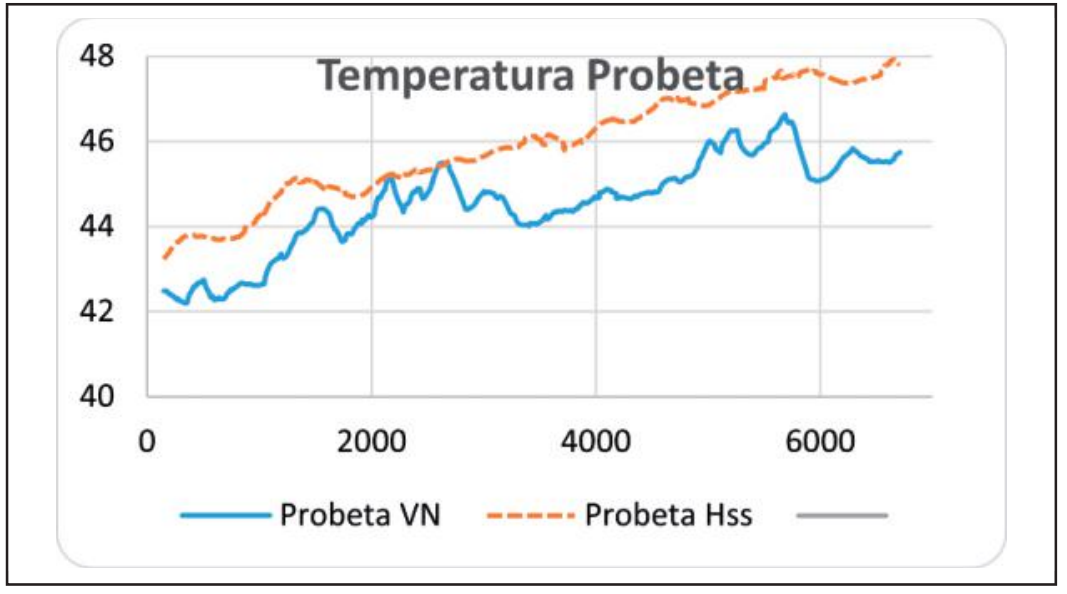

Figura 5. Temperatura en las probetas de acero UNS G1020, durante el cilindrado usando herramientas con y sin recubierto de VN.

Fuente: autores

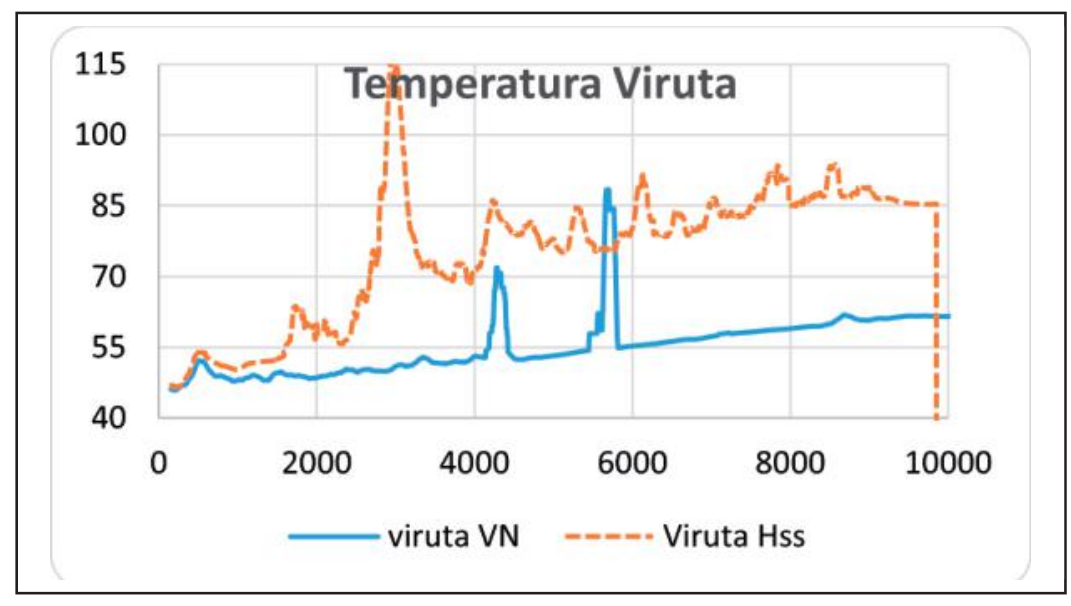

Figura. 6. Temperatura en el material removido,

durante el cilindrado usando herramientas con y sin recubierto de VN.

Fuente: autores 
Al efectuar el análisis de las máximas temperaturas registradas en las pruebas, por una parte se puede observar que las temperaturas más altas en cualquier tipo de herramienta usada siempre se encuentran en el material removido, por otro lado, se determina que al realizar trabajos de mecanizado con herramientas recubiertas estas presentan las temperaturas más bajas, lo que indica que la transferencia energética se da desde la herramienta a la probeta, lo que hace evidenciar que la vidaútil de la herramienta con recubrimiento se verá favorecida. Al analizar los valores de temperatura de las herramientas sin recubrir, se observa que la temperatura es más alta en la herramienta que en el material lo cual reduce considerablemente la vida útil de esta y hace más propenso a la generación del desgaste adhesivo en ella. Todo esto se aprecia mejor en la tabla IV:

Tabla IV. Temperaturas máximas de corte en $\left({ }^{\circ} \mathrm{C}\right)$

\begin{tabular}{|l|c|c|}
\hline \multirow{2}{*}{\multicolumn{1}{c|}{ Medición }} & \multicolumn{2}{c|}{ Tipo de Herramienta } \\
\cline { 2 - 3 } & Nitruro de Vanadio & Sin recubrimiento \\
\hline Herramienta & 42,91 & 71,7 \\
\hline Probeta & 46,59 & 47,65 \\
\hline Material removido & 88,39 & 115,8 \\
\hline
\end{tabular}

Fuente: autores

Con los valores reales y estadísticos se pudo determinar la calidad del recubrimiento aplicado al buril para efectuar trabajos de arranque de viruta a diferentes parámetros de corte de metal. Esto se lograra con ayuda del sensor de temperatura, el cual sirvió como instrumento de obtención de datos reales acerca de la transferencia calorífica entre los tres elementos de estudio que son: viruta, material de trabajo y herramienta de corte, indicando que la transferencia energética se realiza a la viruta por eso se genera superficies con menor rugosidad.
Los recubrimientos aumentan la resistencia al desgaste en las herramientas de corte ya que aumentan la tenacidad en el filo y resistencia a roturas. Debido a que tiene una dureza de $2200 \mathrm{HV}$ y un coeficiente de fricción de 0.4 .

\subsection{Caracterización morfológica de la huella de desgaste}

Culminado el proceso de cilindrado se toma la herramienta para tomar la micrografía en el área de trabajo, esto se aprecia en la Figura 7.

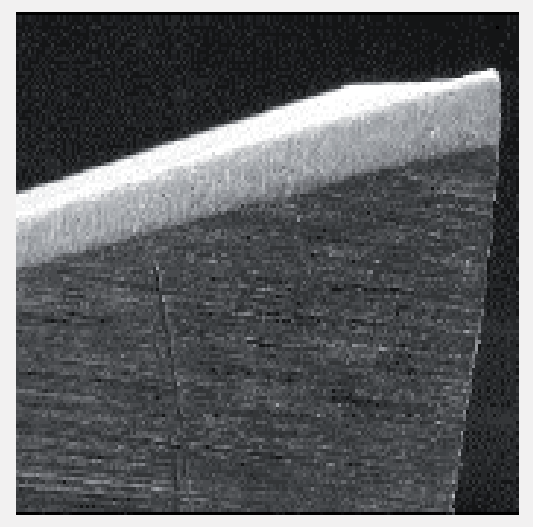

(a)

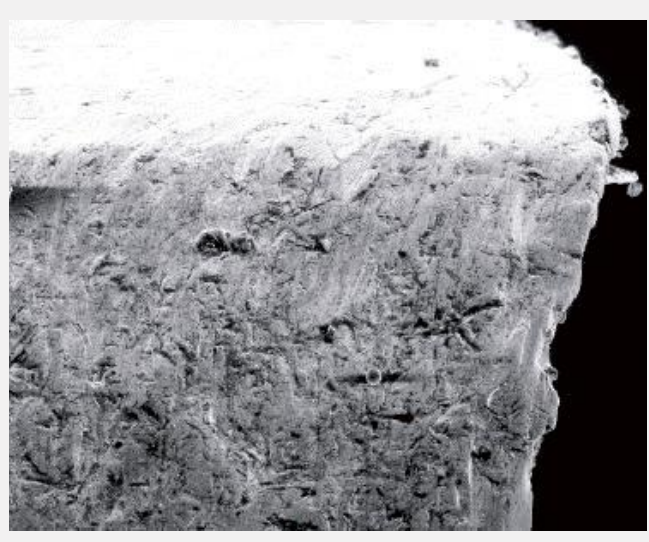

( b )

Figura 7. Micrografías SEM del buril recubierto con VN. Comparación de la huella de desgaste luego de los procesos de mecanizado a) antes de mecanizar con una resolución de $500 \mu \mathrm{m}$ a $34 \mathrm{X}$ b) después de mecanizar con una resolución de $500 \mu \mathrm{m}$ a $34 \mathrm{X}$. Fuente: Autores 
De las imágenes anteriores se observa que el desgaste en la herramienta con monocapa de $\mathrm{VN}$, es menor que el presentado en la herramienta sin recubrimiento, de tal forma da a entender que existió una mejor transferencia energética en el proceso, se asocia a la formación de óxido de Vanadio la cual actúa como lubricante entre las partes en contacto disminuyendo el coeficiente de fricción y a su vez el desgaste. [15]

Sintetizando los corolarios, el uso de recubrimiento de nitruro de vanadio, genera menor transmisión de energía a la probeta, y al haber estabilidad de la transferencia de energía en la zona de operación de la herramienta, el desgaste de este será menor; a menor tasa de cambio de temperatura, es mejor la calidad del proceso de corte y el acabado de la pieza. [16].

\section{Conclusiones}

- Los recubrimientos monocapa de VN que fueron depositados mediante la técnica física de fase vapor (PVD), presentaron mejor desempeño en operaciones de arranque de viruta frente a las otras herramientas de estudio ya que con estas se logra que la herramienta tenga temperaturas más bajas al momento de realizar un corte respecto al material de trabajo.

- El acabado superficial también mejoró notablemente con la implementación del recubrimiento duro de monocapa de nitruro de vanadio presentando una rugosidad de $1,28 \mu \mathrm{m}$, en contraste con el obtenido por la herramienta sin recubrir que fue de $3,0 \mu \mathrm{m}$, esto debido a las propiedades autolubricantes que se ganan a implementar recubrimientos duros.

- Con este trabajo se demostró que los recubrimientos duros aumentan la vida útil de las herramientas de corte por arranque de viruta, ya que trabajan con rangos de temperatura muy por debajo de los trabajados con herramientas convencionales lo cual genera que exista menos probabilidad de evidenciar en este tipo de herramientas el tipo de desgaste más frecuente que es el de tipo adhesivo.

\section{Agradecimientos}

Los autores agradecen a la Vicerrectoría de Investigaciones de la Universidad Militar Nueva Granada por la financiación de este trabajo, derivado del proyecto ING1775 vigencia 2015 y a la Universidad Santo Tomas por el apoyo que dio para el desarrollo de este trabajo.

\section{Referencias}

[1] Y. Morales Tamayo, R. Pérez Rodríguez, P. Zambrano Robledo, et al. "Comportamiento del desgaste del flanco en el torneado en seco de alta velocidad del acero AISI 316L", Ingeniería Mecánica, vol. 16, no. 3, pp.238-245, 2014.

[2] A. Torres, I. Puertas and C. J. Luis. "Surface Roughness Analysis on the Dry Turning of an Al-Cu Alloy." Procedia Engineering, vol, 132, pp. 537-544, 2015.

[3] R. Bulfin and D. Sipper, Planeación y Control de la Producción. 1988.

[4] S. B. Abusuilik. "Pre-, intermediate, and post-treatment of hard coatings to improve their performance for forming and cutting tools", Surface and Coatings Technology, vol. 284, pp. 384-395, 2015.

[5] A. Posso, L. Yate, J. C. Caicedo, et al. "Caracterización de Películas Delgadas de Nitruro de Vanadio (VN) Depositadas por Magnetrón Sputtering D.C," Revista 
de la Sociedad Colombiana de Física, vol. 41, no. 1, pp. 17-19, 2009.

[6] J. J. Olaya Florez and W. H. Muños. "Producción y resistencia a la corrosión de multicapas de nitruro de niobioniobio", Revista Latinoamericana de Metalurgia y Materiales, vol. 33, no. 1, pp. 66-73. 2013.

[7] B. Krause, M. Kaufholz, S. Kotapati, et al. "Angle-resolved X-ray reflectivity measurements during off-normal sputter deposition of VN", Surface and Coatings Technology, vol. 277, pp. 5257, 2015.

[8] M. Folkenant, K. Nygren, P. Malinovskis, eta al. "Structure and properties of $\mathrm{Cr}-\mathrm{C} / \mathrm{Ag}$ films deposited by magnetron sputtering", Surface and Coatings Technology, vol. 281, pp. 184192, 2015.

[9] Q. Luo. "Temperature dependent friction and wear of magnetron sputtered coating TiAlN/VN", Wear, vol. 271, no. 9-10, pp. 2058-2066, 2011.

[10] J. Alami, Z. Maric, H. Busch, et al. "Enhanced ionization sputtering: A concept for superior industrial coatings", Surface and Coatings Technology, vol. 255, no. 25, pp. 43-51, 2014.

[11] Q. Luo, S. Yang and K.E. Cooke, "Hybrid HIPIMS and DC magnetron sputtering deposition of TiN coatings: Deposition rate, structure and tribological properties", Surface and Coatings Technology, vol. 236, pp. 1321, 2013.

[12] N. U. Tovar Quiroz, U. Fuentes Guerrero, $\quad$ C. A. ortiz otalora and E. Vera Lopez. "Estudio electroquímico del acero aisi-sae 1020, nitrurado vía plasma durante tiempos de 10 y 15 horas" Respuestas, vol. 11, no. 2, pp. 27-32, 2006.

[13] J. C. Caicedo, G. Zambrano, W. Aperador, et al. "Mechanical and electrochemical characterization of vanadium nitride (VN) thin films", Applied Surface Science, vol. 258, no. 1, pp. 312-320, 2011.

[14] C. Escobar, M. Villarreal, J. C. Caicedo, et al. "Novel performance in physical and corrosion resistance HfN/VN coating system", Surface and Coatings Technology, vol. 221, pp. 182-190, 2013.

[15] J. C. Caicedo, C. Escobar, W. Aperador, et al. "Heterostructures design for HfNitride/V-Nitride system", Journal of Physics and Chemistry of Solids, vol. 87, pp. 87-94, 2015.

[16] E. C. Corredor Vega, C. A. ortiz otálora, E. V. López and J. E. Alfonso orjuela. "Caracterización en ambientes biológicos del comportamiento a la corrosión de películas delgadas de ti6ai4v crecidas por pulverización catódica rf sobre sustratos de acero 3161 ", Respuestas, vol. 11, no. 2, pp. 3338, 2006.
Jul - Dic 2016

ISSN 0122-820X

E-ISSN 2422-5053 PP: 103-111 